\title{
Determination of the Primality of $N$ by Using Factors of $N^{2} \pm 1$
}

\author{
By H. C. Williams and J. S. Judd
}

\begin{abstract}
Algorithms are developed which can be used to determine the primality of a large integer $N$ when a sufficient number of prime factors of $N^{2}+1$ are known. A test for the primality of $N$ which makes use of known factors of $N-1, N+1$ and $N^{2}+1$ and the factor bounds on these numbers is also presented. In order to develop the necessary theory, the properties of some functions which are a generalization of Lehmer functions are used. Several examples of numbers proved prime by employing these tests are given.
\end{abstract}

1. Introduction. Some of the most effective methods for determining the primality of a large integer $N$ depend upon the knowledge of factors of $N-1$ or $N+1$. For an excellent discussion of many of these techniques see Brillhart, Lehmer and Selfridge [1] and Selfridge and Wunderlich [6]. It may, however, occur that we are more easily able to determine more factors of $N^{2}+1$, than of $N^{2}-1$. For example, if $N=$ $\left(2^{198}+1\right) 41-2^{99}$, then

$$
\begin{aligned}
N-1 & =2^{3} \cdot 53 \cdot 2837 \cdot R_{1}, \\
N+1 & =2 \cdot 3 \cdot R_{2}, \\
N^{2}+1 & =2 \cdot 5^{2} \cdot 13 \cdot 37 \cdot 109 \cdot 397 \cdot 2113 \cdot 42373 \cdot 235621 \cdot 312709 \cdot R_{4},
\end{aligned}
$$

where $R_{1}, R_{2}, R_{4}$ are each composite and any prime factor of $R_{1} R_{2} R_{4}$ exceeds $10^{6}$. This number is a special case of

$$
N=x^{2} b-x+b \quad\left(x=2^{99}, b=41\right) .
$$

For these numbers,

$$
N^{2}+1=\left(x^{2}+1\right)\left(b^{2} x^{2}-2 b x+b^{2}+1\right)
$$

hence, if $x^{2}+1$ can be easily factored, we can find factors of $N^{2}+1$. We also remark here that if $f_{n}$ is the Fibonacci number $\left(\alpha^{n}-\beta^{n}\right) /(\alpha-\beta)$ and $l_{n}$ is the Lucas number $\alpha^{n}+\beta^{n}$, where $\alpha+\beta=-\alpha \beta=1$, then

$$
f_{2 n+1}^{2}+1=f_{2 n-1} f_{2 n+3}, \quad l_{2 n}^{2}+1=5 f_{2 n-1} f_{2 n+1}
$$

thus, many of the Fibonacci and Lucas numbers are examples of numbers $N$ such that $N^{2}+1$ may be fairly easily factored.

The purpose of this paper is to develop algorithms which can be used to determine

Received May 5, 1975.

AMS (MOS) subject classifications (1970). Primary 10A25; Secondary 10A35.

Key words and phrases. Primality testing, Lucas functions, Lehmer functions. 
the primality of $N$ when a sufficient number of prime factors of $N^{2}+1$ are known. We will also develop a combined test for primality which is an extension of that given in Section 7 of [1]. This test makes use of the knowledge of factors of $N-1, N+1$, $N^{2}+1$ and the factor bounds of these numbers in order to determine the primality of $N$. In order to do this, we review some properties of functions introduced by Williams [7] and then show how these functions may be utilized in the development of the desired primality criteria. Finally, we give some examples of numbers which were proved prime by using these algorithms.

2. The Functions $V_{m}$ and $U_{m}$. Let $\rho_{1}, \rho_{2}$ be the two zeros of $x^{2}-P_{1} x+P_{2}$; and let $\alpha_{i}, \beta_{i}(i=1,2)$ be the zeros of

$$
x^{2}-\rho_{i} x+Q \quad(i=1,2)
$$

where $P_{1}, P_{2}, Q$ are integers such that $\left(P_{1}, P_{2}, Q\right)=1$. Put $\delta=\rho_{2}-\rho_{1}$ and define $\Delta=\delta^{2}=P_{1}^{2}-4 P_{2}, E=\left(P_{2}+4 Q\right)^{2}-4 Q P_{1}^{2}$,

$$
V_{n}=\frac{1}{\delta}\left|\begin{array}{cc}
\alpha_{1}^{n}+\beta_{1}^{n} & \rho_{1} \\
\alpha_{2}^{n}+\beta_{2}^{n} & \rho_{2}
\end{array}\right|, \quad U_{n}=\frac{1}{\delta}\left|\begin{array}{cc}
1 & \alpha_{1}^{n}+\beta_{1}^{n} \\
1 & \alpha_{2}^{n}+\beta_{2}^{n}
\end{array}\right|
$$

The first few values for these functions are given in the table below.

$\begin{array}{ccc}n & V_{n} & U_{n} \\ 0 & 2 & 0 \\ 1 & 0 & 1 \\ 2 & -P_{2}-2 Q & P_{1} \\ 3 & -P_{1} P_{2} & P_{1}^{2}-P_{2}-3 Q \\ 4 & P_{2}^{2}-P_{1}^{2} P_{2}+4 P_{2} Q+2 Q^{2} & P_{1}^{3}-2 P_{1} P_{2}-4 P_{1} Q\end{array}$

TABLE 1

Since

$$
\begin{aligned}
& V_{n+4}=P_{1} V_{n+3}-\left(P_{2}+2 Q\right) V_{n+2}+Q P_{1} V_{n+1}-Q^{2} V_{n}, \\
& U_{n+4}=P_{1} U_{n+3}-\left(P_{2}+2 Q\right) U_{n+2}+Q P_{1} U_{n+1}-Q^{2} U_{n},
\end{aligned}
$$

we see that $V_{n}, U_{n}$ are integers for any integer $n \geqslant 0$. It should also be noted that

$$
V_{n+m}=V_{n} V_{m}-P_{2} U_{n} U_{m}-Q^{m} V_{n-m}, \quad U_{n+m}=U_{n} V_{m}+V_{n} U_{m}+P_{1} U_{n} U_{m}-Q^{m} U_{n-m},
$$

for any integers $n, m$.

If $N$ is any integer and $\left(N, Q P_{2}\right)=1$, find $M, S$ such that

$$
Q M \equiv-P_{2} S \equiv 1 \quad(\bmod N)
$$

and put

$$
X_{k} \equiv\left\{\begin{array}{ll}
S^{2} M^{k / 2} V_{k} & (k \text { even }) \\
S M^{(k+1) / 2} V_{k} & (k \text { odd })
\end{array}(\bmod N)\right.
$$




$$
W_{k} \equiv\left\{\begin{array}{ll}
S^{2} M^{k / 2} U_{k} & (k \text { even }) \\
S M^{(k+1) / 2} U_{k} & (k \text { odd })
\end{array}(\bmod N)\right.
$$

From the formulas given above, we see that

$$
\begin{aligned}
& W_{2 m+1} \equiv X_{2(m+1)}+X_{2 m}, \\
& X_{2 m+1} \equiv-P_{2}\left(W_{2 m+2}+W_{2 m}\right)-P_{1} W_{2 m+1}(\bmod N),
\end{aligned}
$$

and

$$
\begin{aligned}
& X_{2 m} \equiv\left\{\begin{array}{ll}
Q\left(X_{m}^{2}-P_{2} W_{m}^{2}\right)-2 S^{2} & (m \text { odd }) \\
P_{2}^{2}\left(X_{m}^{2}-P_{2} W_{m}^{2}\right)-2 S^{2} & (m \text { even })
\end{array} \quad(\bmod N),\right. \\
& W_{2 m} \equiv\left\{\begin{array}{ll}
Q\left(2 X_{m} W_{m}+P_{1} W_{m}^{2}\right) & (m \text { odd } \\
P_{2}^{2}\left(2 X_{m} W_{m}+P_{1} W_{m}^{2}\right) & (m \text { even })
\end{array}(\bmod N)\right.
\end{aligned}
$$

Using these formulas, we can evaluate $W_{k}(\bmod N)$ for any $k>0$ in $O(\log k)$ operations (Lehmer [3]). Since $\left(U_{k}, N\right)=\left(W_{k}, N\right)$, we see that this technique for evaluating $W_{k}(\bmod N)$ may be used in the evaluation of $\left(U_{k}, N\right)$.

3. Properties of $U_{m}$. Several divisibility properties of the functions $U_{n}$ may be deduced from the more general results of [7]. We list here some of the properties that will be needed in later sections of the paper.

We first note that if $n$ and $m$ are positive integers, then $U_{n} \mid U_{m n}$ :

We now require a few definitions. Let the function $U_{n}$ be given by parameters $P_{1}, P_{2}, Q$. For each prime $p$ such that $(p, 2 \Delta E Q)=1$, we associate with $U_{n}$ the functions

$$
\delta(p)=(\Delta \mid p), \quad \epsilon(p)=(E \mid p), \quad \eta(p)=(e(p) \mid p)
$$

where the symbol $(x \mid p)$ is the Legendre symbol,

$$
e(p)=P_{1}^{2}+\Delta-16 Q+2 P_{1} d \quad \text { and } d^{2} \equiv \Delta \quad(\bmod p) .
$$

We see that the function $\eta(p)$ is defined only when $\delta(p)=+1$. We also define the function $\Psi(p)$ by putting

$$
\Psi(p)= \begin{cases}\left(p^{2}-\epsilon\right) / 2 & \text { when } \delta=-1, \\ \left(p^{2}-1\right) / 2 & \text { when } \delta=+1, \epsilon=-1, \\ p-\eta & \text { when } \delta=\epsilon=1\end{cases}
$$

where $\delta=\delta(p), \epsilon=\epsilon(p), \eta=\eta(p)$.

Let $m$ be any integer such that $(m, Q)=1$ and let $U_{\tau_{0}}$ be the first term of the sequence

$$
U_{1}, U_{2}, U_{3}, \ldots, U_{n}, \ldots
$$

in which $m$ occurs as a factor. We define the increasing sequence of integers $\tau_{0}, \tau_{1}$, $\tau_{2}, \ldots, \tau_{j}, \ldots$ by saying $U_{\tau_{j}}$ is the first term of the sequence (*) such that $m \mid U_{\tau_{j}}$ 
and $\tau_{i} \nmid \tau_{j}(i=0,1,2, \ldots, j-1)$. We call these $\tau$ 's the orders of apparition of $m$ and denote them by $\tau_{j}(m)$. We are now able to give the following important theorem.

THEOREM. If $p$ is a prime and $(p, 2 \Delta E Q)=1$, there exists at least one order of apparition of $p$. Further, if $\tau_{j}(p)$ is any order of apparition of $p$, then $\tau_{j}(p) \mid 2 \Psi(p)$.

Proof. This follows as a result of Theorems 6.6, 7.1, and 7.2 of [7].

With this result we easily deduce

THEOREM 1. Let $(N, 2 \Delta Q E)=1$ and $N \mid U_{m}$. If $q$ is any odd prime divisor of $m$ and $N \nmid U_{m / q}$, then any prime divisor $p$ of $N$ which does not divide $U_{m / q}$ must satisfy the congruence

$$
\Psi(p) \equiv 0 \quad\left(\bmod q^{\alpha}\right)
$$

where $q^{\alpha} \| m$.

Proof. Let $\tau$ be an order of apparition of $p$ such that $\tau \mid m$. Clearly, since $p \mid U_{m}$, such a $\tau$ must exist. Now $p \nmid U_{m / q}$; hence, $\tau \nmid m / q$ and, consequently, $\left.q^{\alpha}\right|_{\tau}$. Since $q$ is odd and $\tau \mid 2 \Psi(p)$, the theorem follows.

4. Some Criteria for Primality. In this section we develop some results which will allow us to test an integer $N$ for primality when we know a sufficient number of divisors of $N^{2}+1$. We let the completely factored part of $N^{2}+1$ be denoted by $F_{4}$ and the unfactored part by $R_{4} ; *$ then $N^{2}+1=F_{4} R_{4}$ and $\left(F_{4}, R_{4}\right)=1$.

We select integers $D, C$ such that $(D \mid N)=\left(C^{2}-16 D \mid N\right)=-1$, where the symbol $(X \mid N)$ is the Jacobi symbol. If $H$ and $K$ are integers and

$$
\begin{aligned}
P_{1} & =4\left(2 H^{2}+H K C+2 K^{2} D\right), \\
4 P_{2} & =P_{1}^{2}-16 D, \\
16 Q & =P_{1}^{2}-16\left(H^{2} C+K^{2} C D+8 H K D\right)+16 D,
\end{aligned}
$$

we have

$$
\begin{gathered}
\Delta=16 D, \\
P_{1}^{2}+\Delta-16 Q+2 P_{1} \sqrt{\Delta}=16(H+K \sqrt{D})^{2}(C+4 \sqrt{D}), \\
E=\left(P_{1}^{2}+\Delta-16 Q+2 P_{1} \sqrt{\Delta}\right)\left(P_{1}^{2}+\Delta-16 Q-2 P_{1} \sqrt{\Delta}\right) / 16 \\
=16\left(H^{2}-K^{2} D\right)^{2}\left(C^{2}-16 D\right) .
\end{gathered}
$$

If $p$ is any prime such that $\left(p, 2\left(H^{2}-K^{2} D\right)\left(C^{2}-16 D\right) D\right)=1$ and $(D \mid p)=+1$, then $e(p)=16(H+K \bar{d})^{2}(C+4 \bar{d})$, where $\bar{d}^{2} \equiv D(\bmod p)$. Hence, for $U_{n}$ given by $P_{1}$, $P_{2}, Q$ above, we see for any prime $p$ such that $\left(p, 2\left(H^{2}-K^{2} D\right)\left(C^{2}-16 D\right) D\right)=1$,

$$
\delta(p)=(D \mid p), \quad \epsilon(p)=\left(C^{2}-16 D \mid p\right), \quad \eta(p)=(C+4 \bar{d} \mid p) .
$$

These are all independent of the values of $H, K$; and consequently, we see that the value of $\Psi(p)$ is independent of $H$ and $K$.

For our fixed values of $D$ and $C$ we now define the functions $U_{n}^{(i)}(i=1,2, \ldots)$

*We use the notation $F_{4}$ and $R_{4}$ because $N^{2}+1$ is the fourth cyclotomic polynomial in $N$. 
by using the parameters $P_{1}^{(i)}, P_{2}^{(i)}, Q^{(i)}(i=1,2, \ldots)$, where

$$
\begin{aligned}
& P_{1}^{(i)}=4\left(2 H_{i}^{2}+H_{i} K_{i} C+2 K_{i}^{2} D\right), \\
& P_{2}^{(i)}=4\left(2 H_{i}^{2}+H_{i} K_{i} C+2 K_{i}^{2} D\right)^{2}-4 D, \\
& Q^{(i)}=\left(2 H_{i}^{2}+H_{i} K_{i} C+2 K_{i} D\right)^{2}-\left(H_{i}^{2} C+8 H_{i} K_{i} D+K_{i}^{2} C D\right)+D,
\end{aligned}
$$

and $H_{i}, K_{i}(i=1,2, \ldots)$ are any two integers such that $\left(N, H_{i}^{2}-K_{i}^{2} D\right)=1$.

As it will be necessary to refer to the following statements several times, we put $\bar{F}_{4}=F_{4} / 2$ and put

( $\alpha$ ) For each prime $q \mid \bar{F}_{4}$ there exists some $H_{i}, K_{i}$ such that for the function $U_{n}^{(i)}$

$$
N \mid U_{N^{2}+1}^{(i)} \text { and }\left(U_{\left(N^{2}+1\right) / q}^{(i)}, N\right)=1 .
$$

( $\beta$ ) For some $H_{i}, K_{i}$ we have

$$
N \mid U_{N^{2}+1}^{(i)} \text { and }\left(U_{\left(N^{2}+1\right) / R_{4}}^{(i)}, N\right)=1 .
$$

It should be noted here that, if $N$ is not a divisor of $U_{N^{2}+1}^{(i)}$, then $N$ is composite.

We now describe, by means of the two following theorems, some properties of possible prime divisors of $N$ when either $(\alpha)$ or $(\beta)$ is true. We first give a theorem which is analogous to a recent theorem of Morrison [4] .

THEOREM 2. If $(\alpha)$ is true and $p$ is any prime divisor of $N$, then

$$
\Psi(p) \equiv 0 \quad\left(\bmod \bar{F}_{4}\right) .
$$

Proof. Since $\Psi(p)$ has a value which depends only on the fixed values of $D$ and $C$, it follows that, if $q$ is any prime divisor of $\bar{F}_{4}$ and $(\alpha)$ is true, then $q^{\nu} \mid \Psi(p)$, where $q^{\nu} \| \bar{F}_{4}$; hence, $\bar{F}_{4} \mid \Psi(p)$.

THEOREM 3. If $(\beta)$ is true and all possible prime divisors of $R_{\mathbf{4}}$ are greater than $B_{4}$, then each prime factor $p$ of $N$ must satisfy a congruence of the form

$$
\Psi(p) \equiv 0 \quad(\bmod q),
$$

where $q$ is some prime divisor of $R_{\mathbf{4}}$ depending on $p$.

Proof. Let $\tau=\tau(p)$ be an order of apparition of $p$ such that $\tau k\left(N^{2}+1\right)$; then $\tau \nmid F_{4}$ and, consequently, $\left(R_{4}, \tau\right)>1$. Thus, there must exist a prime $q$ such that $q \mid R_{4}$ and $q \mid \tau$. Since $\tau \mid \Psi(p)$, the theorem follows.

We are now in a position to give the main result of this section.

THEOREM 4. If $(\alpha)$ and $(\beta)$ are both true, all prime factors of $R_{4}$ are greater than $B_{4}$ and $B_{4} F_{4}>N^{2 / 3}+1$, then $N$ is a prime.

Proof. If $p_{i}$ is some prime divisor of $N$, then

$$
\Psi\left(p_{i}\right) \equiv 0 \quad\left(\bmod q_{i} \bar{F}_{4}\right),
$$

where $q_{i}$ is a prime divisor of $R_{\mathbf{4}}$.

Suppose $N=p_{1} p_{2} p_{3} a$ and $a$ is any integer such that $a \geqslant 1$. Since $\Psi\left(p_{i}\right) \mid p_{i}^{2} \pm 1$, $p_{i}^{2} \pm 1$ is even, and $\bar{F}_{4}$ is odd, we have

$$
p_{i}^{2} \geqslant q_{i} F_{4}-1, \quad p_{i}>\sqrt{B_{4} F_{4}-1}
$$


and

$$
N>\left(B_{4} F_{4}-1\right)^{3 / 2} \text {. }
$$

Thus, if $N$ is composite, it must be the product of two distinct primes $p_{1}$ and $p_{2}$.

(Since $(D \mid N)=-1, N$ cannot be a perfect square.) Since $\left(D \mid p_{1} p_{2}\right)=\left(C^{2}-4 D \mid p_{1} p_{2}\right)$ $=-1$, we have

$$
\delta\left(p_{1}\right)=-\delta\left(p_{2}\right), \quad \epsilon\left(p_{1}\right)=-\epsilon\left(p_{2}\right)
$$

Assume $p_{1}$ to be that prime such that $\delta\left(p_{1}\right)=+1$; then $\delta\left(p_{2}\right)=-1$,

$$
p_{2}^{2} \equiv \epsilon\left(p_{2}\right) \quad\left(\bmod q_{2} F_{4}\right) \quad \text { and } \quad p_{1}^{2} \equiv 1 \quad\left(\bmod q_{1} F_{4}\right)
$$

If $F_{4}=2$, we have

$$
p_{1}^{2} \equiv 1 \quad\left(\bmod q_{1}\right) \quad \text { and } \quad p_{2}^{2} \equiv \pm 1 \quad\left(\bmod q_{2}\right) ;
$$

hence,

$$
p_{1} \geqslant 2 q_{1}-1>2 B_{4}-1, \quad p_{2}>\sqrt{2 B_{4}-1},
$$

and $N>\left(B_{4} F_{4}-1\right)^{3 / 2}$.

If $F_{4}>2$, we have

$$
N^{2}=p_{1}^{2} p_{2}^{2} \equiv-1 \quad\left(\bmod F_{4}\right) \quad \text { and } \quad p_{1}^{2} \equiv 1 \quad\left(\bmod F_{4}\right) ;
$$

consequently,

$$
p_{2}^{2} \equiv \epsilon\left(p_{2}\right) \equiv-1 \quad\left(\bmod F_{4}\right)
$$

and $\epsilon\left(p_{2}\right)=-1$. It follows that $\epsilon\left(p_{1}\right)=+1$ and

$$
p_{1} \equiv \pm 1 \quad\left(\bmod q_{1} F_{4}\right)
$$

Putting this result together with

$$
p_{2}^{2} \equiv-1 \quad\left(\bmod q_{2} F_{4}\right)
$$

we see that $N>\left(B_{4} F_{4}-1\right)^{3 / 2}$; thus, $N$ cannot be the product of two or more primes and, therefore, must be a prime.

5. A Further Refinement. If $(\alpha)$ and $(\beta)$ are true and $F_{4}>B_{4} \geqslant 5$, we can lower the bound given in Theorem 4 on $F_{4}$ and still test $N$ for primality. In order to do this it is necessary to show that neither of two cubic equations has three integer roots. This improved result, given as Theorem 5 , is similar to the results obtained in [1] by using the properties of the hyperbola $x^{2}-y^{2}=N$. In order to prove Theorem 5 , it should first be noted that if $f=\epsilon_{1}+r F_{4}$ and $g=\epsilon_{2}+s F_{4}$, where $\left|\epsilon_{1}\right|=\left|\epsilon_{2}\right|=$ 1 and $r, s>B_{4}$, then $f g=\epsilon_{1} \epsilon_{2}+t F_{4}$, where $t>B_{4}$. Thus, if $(\alpha)$ and $(\beta)$ are true and $N$ is composite, we see by results obtained in the proof of Theorem 4 that there exist three integers $k, l, m$ such that

$$
N^{2}=\left(\epsilon_{1}+k F_{4}\right)\left(\epsilon_{2}+l F_{4}\right)\left(\epsilon_{3}+m F_{4}\right),
$$


where $\left|\epsilon_{1}\right|=\left|\epsilon_{2}\right|=\left|\epsilon_{3}\right|=1, \epsilon_{1} \epsilon_{2} \epsilon_{3}=-1$, and $k, l, m>B_{4}$. We also assume here that $(N, 3)=1$.

\section{Theorem 5. Put}

$$
R_{4}=\lambda_{1}+\mu_{1}\left(3 F_{4}\right)+\nu_{1}\left(3 F_{4}\right)^{2} \quad\left(\left|\lambda_{1}\right|,\left|\mu_{1}\right|<3 \bar{F}_{4}\right)
$$

Let

$$
\begin{aligned}
& F_{4} \equiv \gamma \quad(\bmod 3) \quad(|\gamma|=1), \\
& \left(R_{4}+\gamma F_{4}^{2}\right) / 3 \equiv \lambda_{2} \quad\left(\bmod F_{4}\right) \quad\left(\left|\lambda_{2}\right|<\bar{F}_{4}\right), \\
& 1+2 \lambda_{2} \gamma \equiv \theta \quad(\bmod 3) \quad(|\theta| \leqslant 1) \\
& \left(\left(R_{4}+\gamma F_{4}^{2}\right) / 3-\lambda_{2}-\theta F_{4}+(\gamma \theta+\gamma) F_{4}^{2}\right) / 3 F_{4}=\mu_{2}+\nu_{2} F_{4},
\end{aligned}
$$

where $\left|\mu_{2}\right|<\bar{F}_{4}$.

If either of the cubic equations

$$
\begin{gathered}
x^{3}-\lambda_{1} x^{2}-3 \mu_{1} x-9 \nu_{1}=0 \quad\left(\nu_{1} \neq 0\right), \\
x^{3}-3 \lambda_{2} x^{2}-3\left(3 \mu_{2}+\theta\right) x-3\left(3 \nu_{2}-\gamma(\theta+1)\right)+\gamma=0,
\end{gathered}
$$

has three integer roots, then $N$ is composite. If neither of these equations has three integer roots, $N^{2}<C$, where

$$
C=\left(B_{4} F_{4}-1\right)\left(-1+\left(B_{4}-3\left|\lambda_{2}\right|\right) F_{4}+\left(3 \bar{F}_{4}-1\right) F_{4}^{2}\right),
$$

and $(\alpha),(\beta)$ are both true, then $N$ is a prime.

Proof. If (2) has three integer roots $x_{1}, x_{2}, x_{3}$, we have

$$
\begin{gathered}
x_{1}+x_{2}+x_{3}=3 \lambda_{2}, \\
x_{1} x_{2}+x_{2} x_{3}+x_{3} x_{1}=-3\left(3 \mu_{2}+\theta\right), \\
x_{1} x_{2} x_{3}=3\left(3 \nu_{2}-\gamma \theta-\theta\right)-\gamma \neq 0 .
\end{gathered}
$$

Also,

$$
\left(R_{4}+\gamma F_{4}^{2}\right) / 3=\lambda_{2}+\left(3 \mu_{2}+\theta\right) F_{4}+\left(3 \nu_{2}-\gamma \theta-\gamma\right) F_{4}^{2}
$$

hence,

$$
R_{4}=3 \lambda_{2}+3\left(3 \mu_{2}+\theta\right) F_{4}+\left(3\left(3 v_{2}-\gamma \theta-\gamma\right)-\gamma\right) F_{4}^{2}
$$

and

$$
\begin{aligned}
N^{2} & =-1+3 \lambda_{2} F_{4}+3\left(3 \mu_{2}+\gamma\right) F_{4}^{2}+\left(3\left(3 \nu_{2}-\gamma \theta-\gamma\right)-\gamma\right) F_{4}^{3} \\
& =\left(x_{1} F_{4}-1\right)\left(x_{2} F_{4}-1\right)\left(x_{3} F_{4}-1\right)
\end{aligned}
$$

Thus, if (2) has three integer roots, $N^{2}$ has at least three factors greater than 1 ; consequently, $N$ is composite. It can also be shown, by similar reasoning, that $N$ is composite if (1) has three integer roots. 
Suppose now that neither (1) nor (2) has three integer roots, that $(\alpha),(\beta)$ are both true, that $N^{2}<C$, and that $N$ is composite. Then

$$
N^{2}=\left(\epsilon_{1}+k F_{4}\right)\left(\epsilon_{2}+l F_{4}\right)\left(\epsilon_{3}+m F_{4}\right)
$$

where $\left|\epsilon_{i}\right|=1, \epsilon_{1} \epsilon_{2} \epsilon_{3}=-1, k, l, m>B$. Putting $r=\epsilon_{1} \epsilon_{2} m+\epsilon_{2} \epsilon_{3} k+\epsilon_{1} \epsilon_{3} l, s=$ $\epsilon_{1} m l+\epsilon_{2} m k+\epsilon_{3} l k, t=k l m$, we have $R_{4}=r+s F_{4}+t F_{4}^{2}$.

Since $F_{4} \mid N^{2}+1$, we may assume without any loss of generality that $\epsilon_{1}+k F_{4}$ is the square of a prime and that $\epsilon_{1}=-1$. Hence,

$$
\epsilon_{2} m F_{4}+\epsilon_{3} l F_{4}+m l F_{4}^{2} \equiv 0(\bmod 3) \text {. }
$$

From this result we easily deduce that

$$
\epsilon_{2} m F_{4} \equiv \epsilon_{3} l F_{4} \equiv \kappa(\bmod 3),
$$

where $\kappa=0,1$.

Case 1. $\kappa=0$. In this case, we have

$$
s \equiv 0(\bmod 3), \quad t \equiv 0(\bmod 9)
$$

thus,

$$
R_{4}=r+(s / 3)\left(3 F_{4}\right)+(t / 9)\left(3 F_{4}\right)^{2}
$$

and

$$
\lambda_{1} \equiv r\left(\bmod 3 F_{4}\right)
$$

If $r \neq \lambda_{1}$, we have $|r|>3 \bar{F}_{4}$; consequently, one of $k, l, m$ must exceed $\bar{F}_{4}$ and

$$
N^{2}>\left(-1+B_{4} F_{4}\right)^{2}\left(-1+F_{4} \bar{F}_{4}\right)>C .
$$

Thus, $r=\lambda_{1}$ and $s / 3 \equiv \mu_{1}\left(\bmod 3 F_{4}\right)$. If $s / 3 \neq \mu_{1}$, we must have one of $k l, l m$, or $\mathrm{km}$ greater than $3 \bar{F}_{4}$. Hence

$$
N^{2}>\left(-1+B_{4} F_{4}\right)\left(-1+F_{4}\left(B_{4}-\left|\lambda_{1}\right|\right)+3 \bar{F}_{4} F_{4}^{2}\right)>C .
$$

It follows that $r=\lambda_{1}, s / 3=\mu_{1}, t / 9=\nu_{1}$, and we see that (1) must have three integer roots.

Case 2. $\kappa=1$. In this case we have $3|r, 3| s$ and

$$
t=k \operatorname{lm} \equiv-\gamma(\bmod 3) .
$$

Also,

$$
s \equiv 3+2 \gamma r(\bmod 9), \quad t \equiv 2 \gamma+r(\bmod 9) \text {. }
$$

Since

$$
\left(R_{4}+\gamma F_{4}^{2}\right) / 3 \equiv r / 3\left(\bmod F_{4}\right)
$$

we have

$$
r / 3 \equiv \lambda_{2} \quad\left(\bmod F_{4}\right)
$$


If $r / 3 \neq \lambda_{2}$, then $|r|>3 \bar{F}_{4}$, which is not possible; hence,

$$
\begin{aligned}
r / 3 & =\lambda_{2}, \\
s / 3 & \equiv 1+2 \gamma \lambda_{2} \equiv \theta \quad(\bmod 3), \\
(t+\gamma) / 3 & \equiv-\gamma-\gamma \theta \quad(\bmod 3) .
\end{aligned}
$$

Now

$$
\begin{aligned}
\left(\left(R_{4}+\gamma F_{4}^{2}\right) / 3-\lambda_{2}-\theta F_{4}+(\gamma+\gamma \theta) F_{4}^{2}\right) / 3 F_{4} \\
=(s / 3-\theta) / 3+F_{4}((t+\gamma) / 3+\gamma+\gamma \theta) / 3
\end{aligned}
$$

thus,

$$
(s / 3-\theta) / 3 \equiv \mu_{2}\left(\bmod F_{4}\right)
$$

If $\mu_{2} \neq(s / 3-\theta) / 3$, we have

$$
|s / 3-\theta| \geqslant 3 \bar{F}_{4} \text { or }|s / 3| \geqslant 3 \bar{F}_{4}-1 .
$$

One of $\mathrm{kl}, \mathrm{lm}, \mathrm{km}$ must be greater than $3 \bar{F}_{4}-1$ and

$$
N^{2}>\left(B_{4} F_{4}-1\right)\left(-1+\left(B_{4}-3\left|\lambda_{2}\right|\right) F_{4}+\left(3 \bar{F}_{4}-1\right) F_{4}^{2}\right)=C .
$$

Hence,

$$
r / 3=\lambda_{2}, \quad(s / 3-\theta) / 3=\mu_{2}, \quad((t+\gamma) / 3+\gamma+\gamma \theta) / 3=\nu_{2},
$$

and (2) has the three integer roots $\epsilon_{1} \epsilon_{2} m, \epsilon_{2} \epsilon_{3} k, \epsilon_{1} \epsilon_{3} l$. Since this is impossible, $N$ cannot be composite.

6. A Combined Theorem. Let $F_{1}$ be the completely factored part of $N-1$, $F_{2}$ be the completely factored part of $N+1, R_{1}=(N-1) / F_{1}, R_{2}=(N+1) / F_{2}$. For convenience of reference, we give the following tests of [1].

(I) For each prime $p_{i}$ dividing $F_{1}$ there exists an $a_{i}$ such that

$$
a_{i}^{N-1} \equiv 1 \quad(\bmod N) \text { and }\left(a_{i}^{(N-1) / p_{i}}-1, N\right)=1 .
$$

(II) For some $a$,

$$
a^{N-1} \equiv 1 \quad(\bmod N) \quad \text { and } \quad\left(a^{(N-1) / R_{1}}-1, N\right)=1 .
$$

(III) For each prime $q_{i}$ dividing $F_{2}$ there exists a Lucas sequence $\left\{u_{k}^{(i)}\right\}$ with discriminant $D^{\prime}$ for which $\left(D^{\prime} \mid N\right)=-1$,

$$
N \mid u_{N+1}^{(i)} \text { and }\left(u_{(N+1) / q_{i}}^{(i)}, N\right)=1
$$

(IV) For some Lucas sequence $\left\{u_{k}\right\}$ for which $\left(D^{\prime} \mid N\right)=-1$,

$$
N \mid u_{N+1} \text { and }\left(u_{(N+1) / R_{2}}, N\right)=1 .
$$

In [1] the following theorem is proved.

THEOREM. Assume (I), (II), (III), (IV), and suppose all prime factors of $R_{1}$ and $R_{2}$ are respectively $\geqslant B_{1}$ and $B_{2}$. Define $r$ and $s$ by $R_{1}=\bar{F}_{2} s+r\left(0 \leqslant r<\bar{F}_{2}\right)$, and 
let

$$
G=\max \left(B_{1} F_{1}+1, B_{2} F_{2}-1, m F_{1} \bar{F}_{2}+r F_{1}+1\right) \quad(m \geqslant 1) .
$$

Further, in the case that $G=m F_{1} \bar{F}_{2}+r F_{1}+1$, assume $\left(\lambda F_{1} \bar{F}_{2}+r F_{1}+1\right) \dagger N$, $\delta_{0}^{r} \leqslant \lambda<m$, where $\delta_{0}^{r}$ is the Kronecker delta.

If $N<G\left(B_{1} B_{2} F_{1} \bar{F}_{2}+1\right)$, then $N$ is prime.

In this section we will obtain an extension of this theorem which takes into account the factors of $N^{2}+1$ and, to a lesser extent, the factor bound of $R_{4}$. In order to do this we first give some notation.

Put

$$
\begin{aligned}
\bar{F}_{1} & =F_{1} / 2, \quad \bar{F}_{2}=F_{2} / 2, \\
R_{2} & =r+S \bar{F}_{1}, \quad \text { where } 0 \leqslant r<\bar{F}_{1}, \\
S & \equiv k, \quad 2 R_{1} R_{2} \equiv h, \quad h N \equiv g \quad\left(\bmod \bar{F}_{4}\right),
\end{aligned}
$$

where $0 \leqslant k, h, g<\bar{F}_{4}$.

Let $f$ be any unitary divisor of $\bar{F}_{4}$, i.e. $\left(\bar{F}_{4} / f, f\right)=1$. Define

$$
L(f)=-1+r F_{2}+b F_{1} F_{2} \text {, }
$$

where

$$
b \equiv k-f g y \quad\left(\bmod \bar{F}_{4}\right) \quad\left(0 \leqslant b<\bar{F}_{4}\right),
$$

and $(x, y)$ is a solution of the linear Diophantine equation $x\left(\bar{F}_{4} / f\right)-y f=1$. Put $\Lambda=\min _{f \mid \bar{F}_{4}} L(f)$, where the minimum is taken over all unitary divisors of $\bar{F}_{4}$ including 1 and $\bar{F}_{4}$.

LEMMA. If

$$
z \equiv 1 \quad\left(\bmod F_{1}\right), \quad z \equiv-1 \quad\left(\bmod F_{2}\right), \quad z^{2} \equiv-1 \quad\left(\bmod F_{4}\right)
$$

then

$$
z \equiv L(f) \quad\left(\bmod F_{1} \bar{F}_{2} \bar{F}_{4}\right)
$$

for some unitary divisor $f$ of $\bar{F}_{4}$.

Proof. Since

$$
z^{2} \equiv-1 \equiv N^{2} \quad\left(\bmod F_{4}\right)
$$

we have

$$
z \equiv N \quad(\bmod f), \quad z \equiv-N \quad\left(\bmod \bar{F}_{4} / f\right),
$$

for some factor $f$ of $\bar{F}_{4}$. Since $(x-N, x+N) \mid 2 N$ and $\left(2 N, \bar{F}_{4}\right)=1, f$ must be a unitary factor of $\bar{F}_{4}$. Thus,

$$
z \equiv N\left(\bmod f F_{1} \bar{F}_{2}\right), \quad z \equiv-N \quad\left(\bmod \bar{F}_{4} / f\right) .
$$

It follows that

$$
z \equiv N\left(1-\left(N^{2}-1\right) y f\right) \quad\left(\bmod F_{1} \bar{F}_{2} \bar{F}_{4}\right)
$$


where $x\left(\bar{F}_{4} / f\right)-y f=1$. The result follows on noting that

$$
N^{3}-N \equiv g F_{1} \bar{F}_{2} \quad\left(\bmod F_{1} \bar{F}_{2} \bar{F}_{4}\right)
$$

and

$$
N \equiv-1+r F_{2}+k F_{1} \bar{F}_{2} \quad\left(\bmod F_{1} \bar{F}_{2} \bar{F}_{4}\right)
$$

We are now able to give our combined theorem as

TheOREM 6. Assume that $\bar{F}_{4}>1$, (I), (II), (III), (IV), $(\alpha)$ and $(\beta)$ are all true with the value of $D^{\prime}$ used in (III) and (IV) being a square multiple of the value of $D$ used in $(\alpha)$ and $(\beta)$. If $L(f)$ is not a divisor of $N$ for each unitary divisor $f$ of $\bar{F}_{4}$ and $1+h F_{1} \bar{F}_{2} \nmid N$, then $N$ is prime if $N<T$, where $T=\min \left(M_{1} M_{2}, M_{3}^{3}, M M_{3}\right)$ and

$$
\begin{aligned}
M & =1+B_{1} B_{2} B_{4} F_{1} \bar{F}_{2} \bar{F}_{4}, \\
M_{1} & =\max \left(-1+B_{4} F_{4}, 1+B_{1} B_{2} F_{1} \bar{F}_{2}, 1+h F_{1} \bar{F}_{2}+F_{1} \bar{F}_{2} \bar{F}_{4}\right), \\
M_{2} & =\max \left(1+B_{1} F_{1},-1+B_{2} F_{2}, L(1)+m F_{1} \bar{F}_{2} \bar{F}_{4}\right), \\
M_{3} & =\max \left(1+B_{1} F_{1},-1+B_{2} F_{2}, \Lambda+F_{1} \bar{F}_{2} \bar{F}_{4}\right),
\end{aligned}
$$

and $L(1)+t F_{1} \bar{F}_{2} \bar{F}_{4}$ is not a divisor of $N$ for $1 \leqslant t<m$.

Proof. We will say that a prime divisor $p$ of $N$ is of the first kind if $\epsilon(p)=$ $\delta(p)=-1$; otherwise, we call $p$ a prime of the second kind. If $p$ is a prime of the first kind, we must have, by results proved in [1] together with Theorem 2,

$$
p \equiv 1 \quad\left(\bmod F_{1}\right), \quad p \equiv-1 \quad\left(\bmod F_{2}\right), \quad p^{2} \equiv-1 \quad\left(\bmod F_{4}\right) .
$$

Hence,

$$
p \geqslant \max \left(\Lambda+F_{1} \bar{F}_{2} \bar{F}_{4}, 1+B_{1} F_{1},-1+B_{2} F_{2}\right)=M_{3}
$$

If $p$ is a prime of the second kind we have

$$
p \equiv 1 \quad\left(\bmod q_{1} F_{1}\right), \quad p \equiv \pm 1 \quad\left(\bmod q_{2} F_{2}\right), \quad p^{2} \equiv 1 \quad\left(\bmod q_{4} F_{4}\right),
$$

where $q_{i} \mid R_{i}(i=1,2,4)$; thus,

$$
p^{2} \equiv 1 \quad \bmod \left(q_{1} q_{2} q_{4} F_{1} \bar{F}_{2} \bar{F}_{4}\right)
$$

and $p \geqslant \sqrt{M}$. Since $N^{2} \equiv-1\left(\bmod \bar{F}_{4}\right)$ and $\bar{F}_{4}>2$, we must have at least one prime divisor of $N$ which is of the first kind.

If $N$ is the product of three primes, one of them must be of the first kind and since $(D \mid N)=\left(C^{2}-16 D \mid N\right)=-1$, the other two must be of the same kind. Hence, $N \geqslant \min \left(M_{3}^{3}, M M_{3}\right)$, which is impossible.

If $N$ is the product of four or more primes, one is of the first kind, and at least two others must be of the same kind; and we have already seen that this is not possible.

If $N=p_{1} p_{2}$ where $p_{1}, p_{2}$ are distinct primes, we know by the reasoning of Theorem 4, that

$$
p_{1} \equiv 1 \quad\left(\bmod q_{1} F_{1}\right), \quad p_{1} \equiv 1 \quad\left(\bmod q_{2} F_{2}\right), \quad p_{1} \equiv \pm 1 \quad\left(\bmod q_{4} F_{4}\right),
$$


where $q_{i} \mid R_{i}(i=1,2,4)$. It follows that

$$
p_{1} \equiv 1 \quad\left(\bmod q_{1} F_{1}\right), \quad p_{2} \equiv-1 \quad\left(\bmod q_{2} F_{2}\right), \quad p_{2} \equiv \pm N \quad\left(\bmod q_{4} F_{4}\right) .
$$

If $p_{1} \equiv 1\left(\bmod q_{4} F_{4}\right)$, we have

$$
p_{1} \equiv 1 \quad\left(\bmod q_{1} q_{2} q_{4} F_{1} F_{2} F_{4}\right), \quad p_{2} \equiv L\left(\bar{F}_{4}\right) \quad\left(\bmod F_{1} \bar{F}_{2} \bar{F}_{4}\right)
$$

and $N=p_{1} p_{1} \geqslant M M_{3}$. If $p_{1} \equiv-1\left(\bmod q_{4} F_{4}\right)$, we have

$$
p_{1} \equiv 1+h F_{1} \bar{F}_{2} \quad\left(\bmod F_{1} \bar{F}_{2} \bar{F}_{4}\right)
$$

and

$$
p_{1} \equiv 1 \quad\left(\bmod q_{1} q_{2} F_{1} \bar{F}_{2}\right)
$$

consequently,

$$
p_{1} \geqslant \max \left(-1+B_{4} F_{4}, 1+B_{1} B_{2} F_{1} \bar{F}_{2}, 1+h F_{1} \bar{F}_{2}+F_{1} \bar{F}_{2} \bar{F}_{4}\right)=M_{1} .
$$

Also,

$$
p_{2} \geqslant \max \left(1+B_{1} F_{1},-1+B_{2} F_{2}, L(1)+m F_{1} \bar{F}_{2} \bar{F}_{4}\right)=M_{2} ;
$$

and we have $N \geqslant M_{1} M_{2}$. Since $N$ cannot be the product of two or more primes, it must be a prime.

COROllaRY. If the conditions of Theorem 6 are all true except that $M_{1} M_{2}<$ $N<\min \left(M M_{3}, M_{3}^{3}\right)$, then $N$ must be the product of two primes and both of these primes must exceed $\operatorname{Min}\left(M_{1}, M_{2}\right)$. If, on the other hand, we have $N>\min \left(M M_{3}, M_{3}^{3}\right)$, then the smallest prime divisor of $N$ must exceed $\min \left(M_{3}, R\right)$, where

$$
R=\max \left(\sqrt{M}, 1+B_{1} F_{1},-1+B_{2} F_{2}\right) .
$$

Remark 1. We note here that it is an easy matter to factor $N^{2}+1$ by trial division at the same time as $N+1$ and $N-1$. If $d$ is a trial divisor of $N-1$ and leaves a remainder of $r$, then $d \mid N^{2}+1$ if and only if $d \ln (r+2)+2$.

Remark 2. It should be emphasized that it is not always necessary, in determining the primality of a particular $N$, to verify all the assertions (I), (II), (III), (IV), ( $\alpha$ ) and $(\beta)$. For example, if $T=M_{1} M_{2}, M_{1}=1+h F_{1} \bar{F}_{2}+F_{1} \bar{F}_{2} \bar{F}_{4}$ and $M_{2}=L(1)+$ $m F_{1} \bar{F}_{2} \bar{F}_{4}$, it would not be necessary to verify each of (II), (IV) and ( $\beta$ ). For, if $B_{1}>$ $m$, which is usually the case, then $M>M_{2}$ and it would be sufficient to verify (I), (II), (III), and ( $\alpha)$ only.

Remark 3. In practice $T$ is usually $M_{1} M_{2}$ with $M_{1}=1+B_{1} B_{2} F_{1} \bar{F}_{2}$ and $M_{2}=$ $L(1)+m F_{1} \bar{F}_{2} \bar{F}_{4}$. Also, very frequently a simple method of factoring like Pollard's method [5] is successful in finding a fairly large factor of $N^{2}+1$.

Remark 4. In finding a value for $m$ in the theorem, it is not necessary to attempt to divide $L(1)+t F_{1} \bar{F}_{2} \bar{F}_{4}$ into $N$ for each value of $t$ such that $1 \leqslant t \leqslant m$. Since this number must represent a prime factor of $N$, it suffices to divide $N$ by it only when it has no prime factor. For many values of $t, L(1)+t F_{1} \bar{F}_{2} \bar{F}_{4}$ has a small prime factor; when this occurs no trial division by $L(1)+t F_{1} \bar{F}_{2} \bar{F}_{4}$ is required.

Remark 5. Frequently, at least one of the cofactors $R_{1}, R_{2}, R_{4}$ is a pseudoprime. 
Suppose $R_{i}$ is a pseudoprime. Then, if we do not have enough factors of $N^{2} \pm 1$ to demonstrate the primality of $N$, we can attempt to demonstrate the primality of $R_{i}$. If we succeed in this, it becomes a fairly easy matter to verify the primality of $N$. If, on using our theorem, we fail to prove $R_{i}$ a prime, the corollary allows us to find a bound on the largest prime divisor of $R_{i}$. This usually increases the size of $B_{i}$ and very often with this increased value for $B_{i}$ we are able to demonstrate the primality of $N$. (The authors are indebted to John Selfridge for this suggestion.)

7. Some Examples. These tests were implemented on a computer and used to determine the primality of some numbers of special forms. In the following three lists we present some of the primes which were discovered using the tests of Theorems 4 and 5.

For

$$
L=\left(10^{2 n}+1\right) a+10^{n}, \quad M=\left(2^{2 n}+1\right) b-2^{n},
$$

and

$$
N=\left(2^{2 n}+1\right) c+2^{3 n}
$$

some values of $(a, n),(b, n)$ and $(c, n)$ for which $L, M$, or $N$ are prime are given in Tables 2, 3 and 4, respectively.

A computer program was written to implement the algorithm of Theorem 6 on an IBM/370-158 computer. We present below some selected results of running this program.

For

$N=3598020110125739154986036092356326252597494924799183218$

7257385201689,

the sixty-eight digit pseudoprime factor of $f_{353}$ (see Jarden [2]), we have for $B_{1}=B_{2}$ $=B_{4}=4 \times 10^{6}$,

$$
\begin{aligned}
& F_{1}=2^{3} \cdot 3^{3} \cdot 13 \cdot 353 \cdot 6163 \cdot 349291 \\
& F_{2}=2 \cdot 5 \cdot 7 \cdot 1543 \\
& F_{4}=2 \cdot 123757 \cdot 331081
\end{aligned}
$$

For $m>4122$, we have $N<T$, where $T=M_{1} M_{2}, M_{1}=B_{1} B_{2} F_{1} \bar{F}_{2}+1$, and $M_{2}=L(1)+m F_{1} \bar{F}_{2} \bar{F}_{4} . N$ was easily found by the program to be prime.

For

$N=22966686648632120276391228028485200841318497622533370591664502461$, the sixty-five digit pseudoprime factor of $f_{331}$, we have for $B_{1}=B_{2}=B_{4}=3 \times 10^{6}$,

$$
\begin{aligned}
& F_{1}=2^{2} \cdot 3^{2} \cdot 5 \cdot 11 \cdot 331, \\
& F_{2}=2 \cdot 7 \cdot 2137, \\
& F_{4}=2 \cdot 41 \cdot 125813 .
\end{aligned}
$$




\begin{tabular}{lr}
$n$ & \multicolumn{1}{c}{$\boldsymbol{a}$} \\
19 & 3 \\
20 & 11 \\
20 & 161 \\
21 & 77 \\
25 & 21
\end{tabular}

TABLE 2

$\begin{array}{lr}n & b \\ 87 & 9 \\ 87 & 57 \\ 90 & 73 \\ 99 & 41\end{array}$

TABLE 3

$\begin{array}{rr}n & c \\ 83 & 7 \\ 83 & 13 \\ 91 & 31 \\ 91 & 75 \\ 93 & 85 \\ 97 & 15 \\ 97 & 55 \\ 97 & 111 \\ 103 & 13 \\ 103 & 87 \\ 105 & 13 \\ 105 & 109 \\ 107 & 105\end{array}$

TABLE 4

This is not enough information to prove this number prime; however, $R_{1}$ was found to be a pseudoprime.

Now if we put

$N^{\prime}=R_{1}=35043313266550886930317110727341696178275958409675868338467$,

(59 digits)

we find with $B_{1}^{\prime}=B_{2}^{\prime}=B_{4}^{\prime}=3 \times 10^{6}$,

$$
\begin{aligned}
& F_{1}^{\prime}=2 \cdot 3 \cdot 7 \cdot 87631 \cdot 100183, \\
& F_{2}^{\prime}=2 \cdot 2 \cdot 71 \cdot 1093,
\end{aligned}
$$




$$
F_{4}^{\prime}=2 \cdot 5 \text {. }
$$

Here $N^{\prime}>\min \left(M_{3}^{\prime 3}, M^{\prime} M_{3}^{\prime}\right)$; thus, the program determined that any prime divisor of $N^{\prime}$ must exceed

$$
B_{1}^{\prime} F_{1}^{\prime}+1=1106171195598000001
$$

and, consequently, $B_{1}$ for $R_{1}$ can be increased to the value 1106171195598000001 . This, however, is still not enough to prove $N$ prime. It was then discovered that $R_{1}^{\prime}$ is also a pseudoprime.

We put

$$
N^{\prime \prime}=R_{1}^{\prime}=95039484139540488825968859064437770696328870101 \quad \text { (48 digits). }
$$

We find with $B_{1}^{\prime \prime}=B_{2}^{\prime \prime}=B_{4}^{\prime \prime}=3 \times 10^{6}$,

$$
\begin{aligned}
& F_{1}^{\prime \prime}=2^{2} \cdot 5^{2} \cdot 67, \\
& F_{2}^{\prime \prime}=2 \cdot 3^{2} \cdot 53, \\
& F_{4}^{\prime \prime}=2 \cdot 41 \cdot 997 \cdot 1519313 .
\end{aligned}
$$

Then

$$
M_{1}^{\prime \prime} M_{2}^{\prime \prime}<N^{\prime \prime}<\min \left(\left(M_{3}^{\prime \prime}\right)^{3}, M^{\prime \prime} M_{3}^{\prime \prime}\right)
$$

The program verified that any prime divisor of $N^{\prime \prime}$ must exceed $M_{2}^{\prime \prime}$, which has the value

$$
L^{\prime \prime}(1)+F_{1}^{\prime \prime} \bar{F}_{2}^{\prime \prime} \bar{F}_{4}^{\prime \prime}=309165997822073801
$$

thus, we can now increase the size of $B_{1}^{\prime}$ to $3 \times 10^{17}$. Using this value for $B_{1}^{\prime}$, the program found $N^{\prime}$ and then $N$ to both be primes.

We also used Pollard's method to attempt to factor $R_{4}^{\prime \prime}$ and this produced the additional prime factor 565909422161 ; this together with the previous factors was enough for the program to determine $N^{\prime \prime}$ a prime.

At the suggestion of $J$. Selfridge, the number

$$
\begin{aligned}
N & =32656499591185747972776747396512425885838364422981 \quad \text { (50 digits) } \\
& =\sum_{k=1}^{41}(-1)^{k-1} k !
\end{aligned}
$$

was run on the computer. For $B_{1}=B_{2}=B_{4}=2 \times 10^{6}$, we have

$$
\begin{aligned}
& F_{1}=2^{2} \cdot 5 \cdot 13 \cdot 37, \\
& F_{2}=2 \cdot 3 \cdot 41, \\
& F_{4}=2 .
\end{aligned}
$$

This is not enough to prove $N$ a prime; however, $R_{2}$ is a pseudoprime. Putting $N^{\prime}=R_{2}=132749998338153447043807916245985471080643757817$ (48 digits) 
and $B_{1}^{\prime}=B_{2}^{\prime}=B_{4}^{\prime}=2 \times 10^{6}$, we get

$$
\begin{aligned}
& F_{1}^{\prime}=2^{3} \cdot 3 \cdot 167 \cdot 3593, \\
& F_{2}^{\prime}=2 \cdot 1307 \\
& F_{3}^{\prime}=2 \cdot 5 \cdot 61 \cdot 614177 .
\end{aligned}
$$

Hence

$$
M_{1}^{\prime} M_{2}^{\prime}<N^{\prime}<\min \left(M_{3}^{\prime 3}, M^{\prime} M_{3}^{\prime}\right)
$$

and

$$
\begin{aligned}
M_{2}^{\prime} & =L^{\prime}(1)+F_{1} \bar{F}_{2} \bar{F}_{4}=\min \left(M_{1}^{\prime}, M_{2}^{\prime}\right) \\
& =4964870743200170113 .
\end{aligned}
$$

Thus, any prime divisor of $N^{\prime}$ must exceed $M_{2}^{\prime}$ and $B_{2}$ can now be increased to $4.9 \times$ $10^{18}$. With this new value of $B_{2}$, the program was able to prove $N$ prime.

8. Acknowledgments. The authors gratefully acknowledge the suggestions given them by John Selfridge and John Brillhart. They also wish to thank J. Brillhart for making available to them a preprint of [1].

Department of Computer Science

University of Manitoba

Winnipeg, Manitoba, Canada R3T 2N2

1. JOHN BRILLHART, D. H. LEHMER \& J. L. SELFRIDGE, "New primality criteria and factorizations of $2^{m} \pm 1$," Math. Comp., v. 29, 1975, pp. 620-647.

2. DOV JARDEN, Recurring Sequences, 3rd ed., Riveon Lemathematika, Jerusalem, 1973, pp. 41-59.

3. D. H. LEHMER, "Computer technology applied to the theory of numbers," Studies in Number Theory, Math. Assoc. Amer.; distributed by Prentice-Hall, Englewood Cliffs, N. J., 1969, pp. 117-151. MR 40 \#84.

4. M. A. MORRISON; "A note on primality testing using Lucas sequences," Math. Comp., v. 29, 1975, pp. 181-182.

5. J. M. POLLARD, “Theorems on factorization and primality testing," Proc. Cambridge Philos. Soc., v. 76, 1974, pp. 521-528.

6. J. L. SELFRIDGE \& M. C. WUNDERLICH, "An efficient algorithm for testing large numbers for primality," Proc. Fourth Manitoba Conf. on Numerical Math., Winnipeg, Manitoba, 1974, pp. 109-120.

7. H. C. WILlIAMS, “A generalization of Lehmer's functions," Acta Arith. (To appear). 